\title{
Evaluating the Optimal Placement of BINARY SENSORS
}

\author{
Stephen P. Emmons and Farhad Kamangar \\ The University of Texas at Arlington, Arlington, Texas, USA \\ stephen.emmons@mavs.uta.edu and kamangarecse.uta.edu
}

\begin{abstract}
Binary sensor arrays have many applications for detecting and tracking the location of an object. Many considerations factoring into the optimal design for such a system, including the accuracy of location, the extent of the sensor coverage, and the number and size of the sensors used. In this paper, we frame the problem by providing several definitions for sensor coverage and spatial probability for the general case, with special attention to sensor arrays in one dimension as a simplification for the purpose of clarity. We then propose a metric for measuring the overall the utility of a given set of choices that may be used in any algorithm for seeking an optimal outcome by maximizing this utility. Finally, we perform a numerical analysis of several variations of the the proposed metric, again in one dimensions, to illustrate its fitness for the task.
\end{abstract}

\section{KEYWORDS}

Binary Sensors, Optimization

\section{INTRODUCTION}

Binary sensor arrays have been used in many applications for detecting the presence of an object and tracking its location. Examples include finding the current location of an RFID tag within a warehouse and tracking it as it moves, detecting the presence of a harmful chemical or radioactive agent, or finding an intruder in a secure facility using motion detectors.

In each application, there may be many factors that are involved in designing an optimal sensor array. Some applications may require a high degree of accuracy for the location of an object, but not $100 \%$ coverage. Other applications may only be concerned with ensuring that $100 \%$ coverage exists, but not with knowing exactly where an object is located.

In this study, we propose a technique for balancing many factors that go into the design of a sensor array. We consider the precision with which we can take measurements, the coverage we achieve, and the costs associated with a given set of choices.

We start with a brief survey of related work, followed by a precise definition of sensor coverage, the specific characteristics of a one-dimensional sensor array, and an explanation of how we will treat probability. Next we propose a utility function for evaluating the relative merits of various sensor arrays, and analyze several one-dimensional sensor placement schemes using it.

\section{RELATED WORK}

The problem of optimal sensor placement have been covered extensively in the literature. Many DOI : 10.5121/ijist.2013.3101 
different criteria have been put forth as the basis for defining optimality. Often, the actual placement of sensors is of less importance than what information may be derived from them given a variety of assumptions.

Chakrabarty et al. [4] focused attention on how to maximize coverage with a minimum of sensors. Their approach imposed a grid on a two- or three-dimensional field and uniquely identified each grid location with a single sensor.

Aslam et al. [3] dealt primarily with tracking objects in binary sensor arrays using techniques for approximating object location based on proximity to nearby sensors. They took a minimalist view, assuming little or no control over the placement of the sensors.

Shrivastava et al. [9] [8] looked at how $\mathrm{n}$ two-dimensional, omnidirectional binary sensor regions $\mathrm{R}$ partition the sensor field into a maximum of $n^{2}-n+2$ "localization patches" $F$ where $|F| \leq$ $|R|$. This study did not consider any specific arrangements of the sensors, but instead used a generic sensor density $\rho$ as the mean distance among the sensors. They showed that the precision $\Delta$ with which the location of some object within the sensing field is $1 / \rho R$ and that it improves (decreases in size) linearly as either $\rho$ or $R$ increase in size.

Sharif et al. [7] analyzed the cost effectiveness of directional sensors covering a large field. They employed a range of cost functions applied to sensor arrays of differing sizes in an effort to determine the optimal configuration.

Krause et al. [5] delved extensively into the fundamentals of two-dimensional temperature sensor placement, considering aspects of variable sensor region size, sensor placement cost, and optimization of sensor placement using information theory. They showed that optimal placement of sensors is an NP-Hard problem and define optimization as based on a single criteria such as maximal information gain from the system. As a result, they gave special attention to algorithms to reduce the computational complexity of the optimization problem.

More recently, Azadzadeh et al. [2] focused on how to maximize the number of unique "localization patches" found in two-dimensional, omnidirectional sensor arrays, and extended this analysis to directional sensors as well. They did not consider the role of sensor coverage in the sensor arrangement, focusing instead on the internal characteristics of the arrangement alone.

The contribution of this paper is to directly address the question of sensor coverage and combine this along with other criteria into an overall measure of utility of a sensor array.

\section{Defining Sensor Coverage}

We define sensor coverage as the portion of a field $A$ over which we array a set of $n$ sensor regions $R=\left\{r_{1} \ldots r_{n}\right\}$. The sensor regions partition $A$ to define a "sensed" portion of the overall field

$S=\mathrm{U}(R)$. The remaining "un-sensed" portion being $S$, such that $A=S+(\sim S)$. Further, an object known to be in $A$ must exist either in $S$ or $\sim S$. Figure 1 shows an example in two-dimensions where $L_{1}$ and $L_{2}$ represent locations that fall inside and outside of $S$ respectively. 


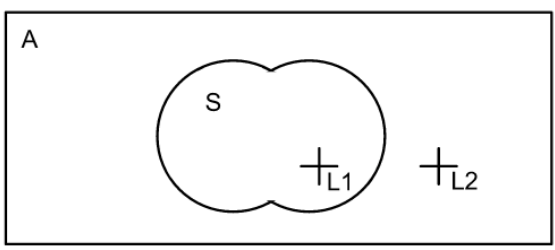

Figure 1: Sensor coverage model

Since we know that sensor regions may overlap, we define a partitioning function $\Phi$ that produces a set of $m$ "patches" $F$ where $\Phi(R)=F=\left\{f_{1} \ldots f_{m}\right\}$. Because these "patches" exactly cover the same portion of $A$ as the sensor regions $R$, it follows that $S=\bigcup(F)$.

Argawal and Sharir [1] showed that $m \leq O\left(n^{d}\right)$ where $d$ is the dimensionality of $A$, and that $m \leq n^{2}$ $-n+2$ in two dimensional case. Therefore, for any set of sensor regions $R$ there is a maximum possible value for $m$ or $\mathbf{m}_{\text {max }}$. Figure 2 illustrates $R$ and $F$ in two dimensions where $n=2$ and $m=$ 3.

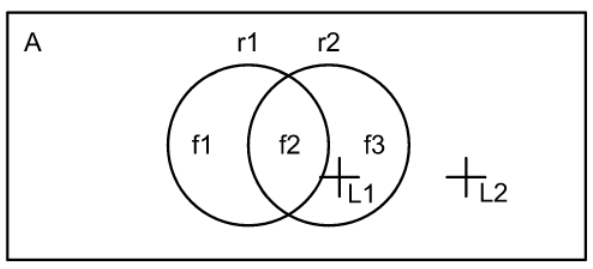

Figure 2: Two sensor regions creating three patches

\section{SENSOR PlaCEMENT In ONe Dimension}

Commonly, we see examples of sensor placement in two dimensions, but by considering binary sensor placement in one dimension, we reduce the complexity involved in studying possible scenarios. Given two sensor regions $r_{1}$ and $r_{2}$, we see in Figure 3 (a), (b), and (c) how they can overlap to produce one, three, or two patches, respectively.
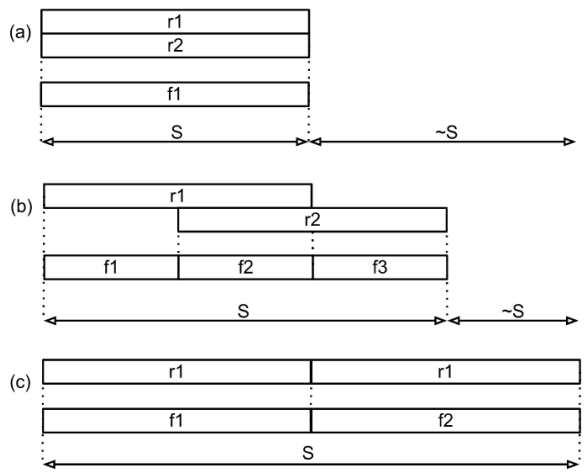

Figure 3: Two sensors (a) at the same location, (b) partially overlapped,and (c) fully covering the field

These examples let us examine the concept of average sensor density. Shrivastava et al. [9] [8] define sensor density $\rho$ as the number of sensor regions covering a given location. In Figure 3 (c), we see that the total field of $A$ is completely covered by $S$ with no overlap among the sensor 
regions $R$. This represents a sensor density of 1 . Figure 3 (a) and (b) likewise have an average sensor density of 1 , but have local sensor densities of 2 where the sensor regions overlap, and 0 where there is no coverage.

These examples also show how the maximum number of patches for 2 sensor regions is 3 . In the general case, the maximum number of "patches" in one dimension is $m \leq 2 n-1$ based on the theoretical limit of $O\left(n^{d}\right)$ where $d=1$.

\section{Clarifying Sensor Probability}

The probability of an object being detected by a sensor array may be defined as either temporal or spatial. Temporal probability concerns the likelihood of an object occurring in $A$ over time, and subsequently, being detected in $S$. For the purpose of this analysis, we focus on spatial probability which deals with the likelihood that an object known to exist in $A$ falls within $S$.

In the spatial case, the probability that such an object falls in $S$ is simply $P(S)$, and outside of $S$ or $\sim S$ is $P(S)$. Given this, the following are true of $S, \sim S$, and $F$ :

$$
\begin{aligned}
& P(S)+P(\sim S)=1 \\
& \sum_{i=1-m} P\left(f_{i}\right)=P(S) \\
& \sum_{i=1-m} P\left(f_{i} \mid S\right)=1
\end{aligned}
$$

These hold regardless of the spatial probability distribution of objects occurring in $A$. In the general case, we calculate the probability for a region $r$ by using the continuous distribution function $(c d f)$ of $A$ and integrate over the range defined by $r$. As an example in one dimension, we calculate $P(r)$ as follows:

$$
P(r)=\int_{\text {rright-rleft }} c d f(x) d x
$$

We may likewise produce $\mathrm{P}(\mathrm{S})$ by summing the probabilities of each "patch" fi $\in \mathrm{F}$ as follows:

$$
P(S)=\sum_{i=1-m} \int_{\text {iright-ileft }} c d f(x) d x
$$

If an object may be found with equal probability throughout $A$, then its probability is a simple proportion of the sub-region size to the overall size of $A$. To facilitate this, we define the function $\Psi$ to produce a set of scalar values $D$ where $\Psi(R)=D=\left\{d_{1} \ldots d_{n}\right\}$. Additionally, we define the related function $\psi$ such that $\forall r_{i} \in R$ and $d_{i} \in \mathrm{D}$ then $\psi\left(r_{i}\right)=d_{i}$. In one dimension, $\psi$ simply returns the sensor region width, whereas in two dimensions, it would returns the region area. With $\psi$, we calculate $P(S)$ and "patch" probability $P(f)$ as follows:

$$
\begin{aligned}
& \forall f \in F, P(f)=\psi(f) / \psi(\mathrm{A}) \text { and } P(f l S)=\psi(f) / \psi(S) \\
& P(S)=\psi(S) / \psi(A)
\end{aligned}
$$

For the remainder of this analysis we limit ourselves to uniform spatial distribution.

\section{Measuring Sensor Array Performance}

In practice, the engineering design of a sensor system is intended to optimize several objectives 
such as cost, efficiency, and accuracy. One way to determine if these objectives are met is to measure each factor and combine them together in a utility function. By maximizing such a function we can find the optimal design choices.

We propose a utility function $U$ that is a linear combination of three weighted objective functions, which are 1) $\Theta$ measuring precision, 2) $\Gamma$ measuring coverage, and 3) $\Omega$ measuring the intrinsic properties of the sensors represented by $R$. Each is paired with a weighting coefficient $W=\left\{w_{1}\right.$, $\left.w_{2}, w_{3}\right\}$ for balancing the relative value of the objectives. In practical situations, the actual values of $W$ depend on factors such as cost of the sensors, required precision, and the consequences of missing an event due to lack of coverage.

$$
U(A, R, W)=w_{1} \Theta(A, R)+w_{2} \Gamma(A, R)+w_{3} \Omega(A, R)
$$

The structure of $U$ is simple with the real work taking place in the objective functions $\Theta, \Gamma$, and $\Omega$, as discussed in the following sections.

\subsection{Considering Sensor Precision}

The concept of "patches" $F$ [2] more precisely identifies the location of a sensed object detected in $S$. Thus we may measure the precision of $R$ in terms of $F$. For this analysis, we examine three definitions of the objective function $\Theta$ for measuring precision, labelled $\Theta_{A}, \Theta_{B}$, and $\Theta_{C}$.

Shrivastava et al. [9] [8] argues for using the Lebesque norm $L_{\infty}$ on $F$ as a worst-case measure of precision, so we base $\Theta_{A}$ on this idea. The intuition is that we can reliably expect location precision to be better than the size of the largest "patch" in $F$. Thus precision increases as $L_{\infty}$ decreases where $L_{\infty}=\max (\Psi(F))$.

To calculate $L_{\infty}$ and for subsequent uses, we need to convert the multi-dimensional regions and sub-regions of $A$ into scalar values.

For this and the rest of the objective functions, we want the values to be normalized and range from 0 to 1 . Recognizing that $0 \leq \max (\Psi(F)) \leq \max (\Psi(R))$, we define $\Theta_{A}$ as follows:

$$
\Theta_{A}=(\max (\Psi(R))-\max (\Psi(F))) / \max (\Psi(R))
$$

Next, we look to the concept of "mean squared error" to find the average accuracy for locations detected within $R$ which also decreases as the precision increases. With this idea, we define $\Theta_{B}$ using $\psi$ to average the square of each "patch" size and scale it as with $\Theta \mathrm{A}$.

$$
\Theta_{B}=\left(\max (\Psi(R))^{2}-\sum_{i=1-m} P\left(f_{i} \mid R\right) \psi\left(f_{i}\right)^{2}\right) / \max (\Psi(R))^{2}
$$

Note that the full equation for "mean squared error" would include the squaring of half the size of each "patch," or a constant $1 / 4$ factor, that is here simply removed to maintain the normalized result from 0 to 1 .

For the definition of $\Theta_{C}$, we look to Shannon entropy [6] as a means of maximizing the "information flow" from the sensor regions $R$. Although it is not properly a measure of precision, entropy's measure of information provides a useful objective function $\Theta_{C}$ to use in contrast to the others. We calculate the entropy $H_{S}$ by treating $F$ as a set of symbols and the probability distribution of an object being detected in any "patch" given its occurrence in $S$.

$$
H_{S}=-\sum_{i=1-m} P\left(f_{i} \mid S\right) \log _{2}\left(P\left(f_{i} \mid S\right)\right)
$$


To understand this, the two binary sensors in Figure 2 may have states 00, 10, 11, and 01 where 1 signifies detecting an object, 0 otherwise. States 01,11 , and 10 would represent the "patches" $f_{1}$, $f_{2}$, and $f_{3}$, respectively. These possible states represent "symbols" each with a probability of occurrence.

Intuitively, we can imagine $H_{S}$ reaching its maximum when the arrangement of sensors $R$ results in "patches" $F$ with an equal likelihood of detecting an object in $S$. Since $H_{S}$ naturally increases from 0 , we define $\Theta_{C}$ simply as $H_{S}$ by the maximum possible value for any $R$. This maximum occurs when $\Phi(R)$ produces an $F$ with the number of "patches" previously denoted as $\mathbf{m}_{\max }$. Thus, $H_{\text {Smax }}=\log _{2}\left(\mathbf{m}_{\text {max }}\right)$.

$$
\Theta_{C}=H_{S} / H_{\operatorname{Smax}}
$$

\subsection{Considering Sensor Coverage}

Our objective function $\Gamma$ provides a measure of sensor coverage. Since we want a value ranging from 0 to 1 , we conveniently chose the the spatial probability of an object known to be in $A$ that is also in $S$, namely $P(S)$.

\subsection{Considering Intrinsic Sensor-Array Properties}

The purpose of our objective function $\Omega$ is to bring characteristics of the sensor array, regardless of the specific arrangement of sensor regions $R$, into $U$. Intuitively, we know that the size and number of the sensors must have some impact on our overall engineering design. At the very least, we would expect the cost of the solution to increase with the number of sensors.

Since we have limited ourselves to uniform sensor region size, we define $\Omega$ as a simple function of sensor count which we want to maximize with the fewest number of sensors. To do this, we use $1 / n$ which is largest when the $n=1$ and is undefined - and uninteresting - for sensor counts of 0 .

\section{Studying Sensor Placement}

With $U$, we have a metric for measuring the combined benefits of higher precision, higher coverage, and fewer sensors across many scenarios. And while we might use this approach to study either changes of field $A$ or weights $W$ for a given sensor array $R$, our primary concerns for the rest of this paper are scenarios where $A$ and $W$ are fixed and only the placement of the sensor regions $R$ varies, the goal being to determine arrangements of $R$ with maximal utility.

It is important to note that by varying only sensor placement, the sensor count remains constant as does the value of $\Omega$. So that going forward, we only consider precision and coverage as defined by $\Theta$ and $\Gamma$.

To facilitate our analysis, we derive three variations of utility functions $-U_{A}, U_{B}$, and $U_{C}$ - based on the three options for $\Theta$ and choosing weights $W=\{1,1,0\}$ to explicitly eliminate $\Omega$.

$$
\begin{aligned}
& U_{A}=\Theta_{A}+\Gamma \\
& U_{B}=\Theta_{B}+\Gamma \\
& U_{C}=\Theta_{C}+\Gamma
\end{aligned}
$$


Starting with two sensor regions at the same location as in Figure 3 (a), we may move them apart to a point where they form three equal "patches" as in Figure 3 (b) and continue until they are fully separated with no overlap as in Figure 3 (c). By setting the sensor width to 1, the width of the overall field to 2 , and computing values for $\Theta_{A}, \Theta_{B}, \Theta_{C}$, and $\Gamma$, we can see that they achieve maximal values in different ways. Figure 4 plots each of these values on the Y-axis with the spacing from 0 to 1 on the $\mathrm{X}$-axis.

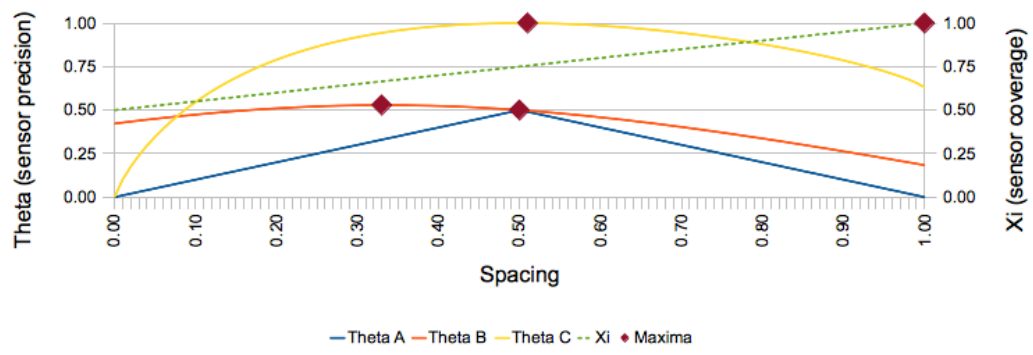

Figure 4: Objective functions for two sensors moving from co-location to full separation

Clearly from this example, we see that any choice for $\Theta$ or $\Gamma$ by itself may give a different maximal result. The "best" choice for coverage $\Gamma$ occurs at full separation of the sensor regions, while the "best" choices for precision $\Theta$ lie elsewhere with no clear criteria for choosing one or the other. By combining $\Theta$ and $\Gamma$ to produce our three derived versions of $U$ as in Figure 5, we see how that the overall utility is often maximally different than either taken separately.

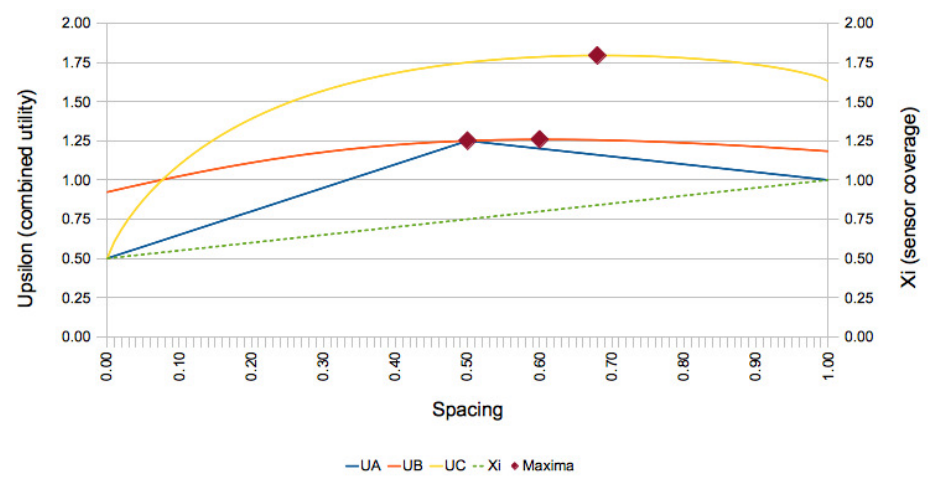

Figure 5: Derived utility functions for two sensors moving from co-location to full separation

\section{COMPARING EVEN AND RANDOM SPACING}

Much of the literature assumes random placement of binary sensors in its analysis. Of course for well-known or fixed fields $A$, we may place the sensors in specific locations in an effort to maximize their effectiveness. For this study, we contrast random sensor placement with a continuation of the evenly-spaced sensor placment approach from the previous section.

To test this idea, we define an overall field width of 100 and sensor regions of uniform width of 1 , such that $\Phi(\mathrm{A})=100$ and $\forall r \in R, \psi(r)=1$. Next, we create scenarios with sensor densities of $\rho=1.0$ and 2.0, resulting in values of $n=100$ and 200, respectively. For each sensor density $\rho$, we then evenly space the sensor regions from 0 to 1 by increments of 0.001 , and also generate 100 random scenarios where the placement of each may occur anywhere within $A$. Finally, we 
plot our three measures of sensor precision $\Theta$ against the percentage of coverage $A$ for each value of $\rho$ in Figure 6 and Figure 7.

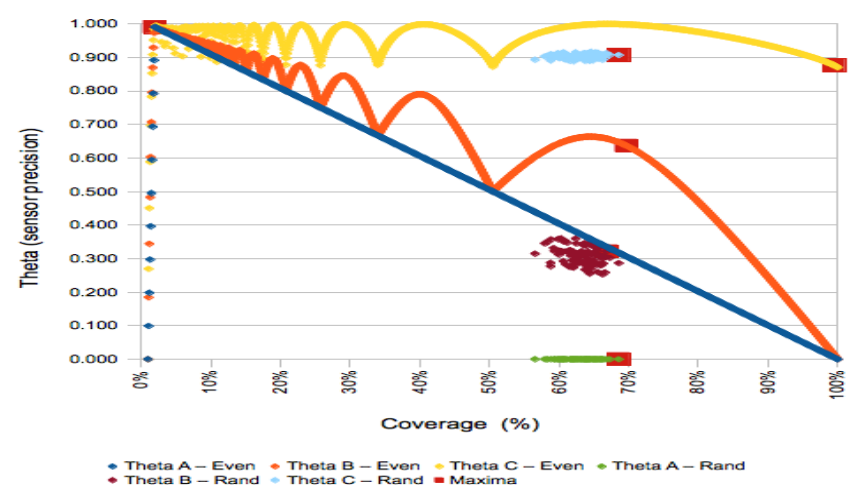

Figure 6: Plot of precision objectives $\Theta$ against percent coverage of $A$ with density $\rho=1.0$ and indicating maximum utility $U$ for each

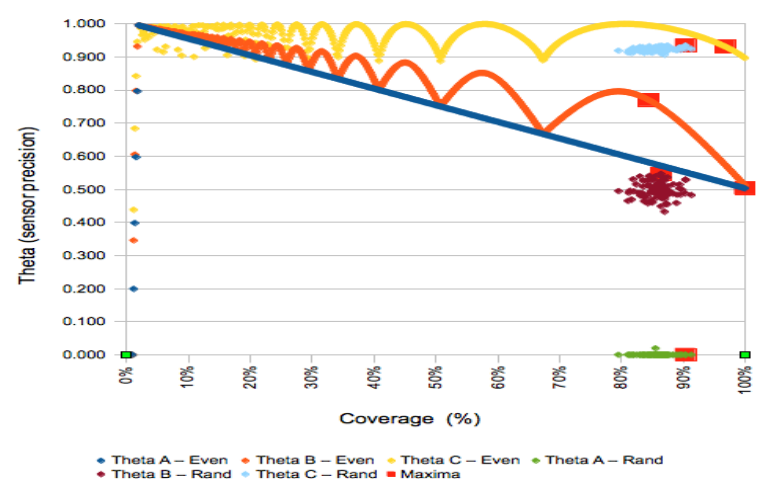

Figure 7: Plot of precision objectives $\Theta$ against percent coverage of $A$ with density $\rho=2.0$ and indicating maximum utility $U$ for each

We are able to make several important observations from these numerical studies. Perhaps the most significant is that even spacing produces better results than random spacing in almost all cases, and that some evenly-spaced placement option is always superior to the best random result. As we might expect, the random results tend to cluster around an average coverage range that increases with $\rho$. Whereas, the even spacing results for each variation of $\Theta$ display a characteristic pattern of their underlying approach to measurement.

Another important observation is the location of the maximum utility function $U$. For example, $U_{B}$ in Figure 6 achieves its maximum with only $70 \%$ coverage, given $W=\{1,1,0\}$.

With even spacing, all measures display low values for the smallest possible coverage where spacing is 0 and all sensor regions $R$ are co-located and have a coverage of $\max (\Psi(R)) / \psi(A)=$ 0.1 . The measures quickly reach their maximum at spacing of $\max (\Psi(R)) / n$ where the uniform sensor size is divided by sensor count to produce exactly $n$ "patches" of equal size.

When looking specifically at objective function $\Theta_{A}$, we see that even spacing shows a linear decrease in precision as the largest "patch" increases in size to $\psi(A) / n$ when $R$ achieves full coverage. However, multiple evaluations using random spacing almost always produced at least 
one "patch" based on an isolated sensor region such that $\max (\Phi(F))=1$, effectively making all results the same. With even spacing, result is not very different in practical terms since the mostly-linear relationship between $\Theta_{A}$ and $\Gamma$ leads to either choosing the maximum precision or maximum coverage option, and which choice is highly sensitive the weights $W$ used.

Both $\Theta_{B}$ and $\Theta_{C}$, display an interesting pattern of local minima and maxima for the even- spacing scenarios. By closely inspecting the data, we see that the local minima occur when sensor boundaries align such that the number of "patches" generated for the arrangement drops significantly and/or result in high variance of size. Despite the differences in calculation, both approaches generate local minima for the same spacing intervals, with local maxima often at different spacing intervals.

$\Theta_{B}$ based on the idea of average accuracy generally decreases as coverage increases despite the local fluctuations, which we would intuitively expect since the "patch" sizes are generally getting larger and the precision therefore must suffer. On the other hand, $\Theta_{C}$ based on entropy suggests the idea that many spacing choices may result in near-maximal information flow.

Not all of the local maxima displayed for $\Theta_{C}$ are, in fact, equal as would be evident by closely inspecting the data, but they are, nevertheless, quite close for very different even-spacing configuration. This quality tends to result in the spacing whose local maxima for $\Theta_{C}$ is also closest to full coverage being selected as having the maximum utility for the $U_{C}$ variant. Intuitively, $U_{C}$ leads us to a desirable outcome where coverage is high, if not full, but the "best" choice takes into consideration the performance of that coverage as a source of information.

Overall, the $U_{A}$ variant of utility does not effectively assist in balancing the considerations of precision and coverage, while both, $U_{B}$ and $U_{C}$ lead to maximum results that balance both.

\section{CONCLUSIONS AND FUture WORK}

By studying the utility function $U$, we find that the variations using "mean squared error" $\left(\Theta_{B}\right)$ and entropy $\left(\Theta_{C}\right)$ show promising results as a way to balance the competing factors of precision and coverage in the design of a sensor array.

Additional work is necessary to apply this method to specific sensor array designs, as well as the selection of different weighting factors $\mathrm{W}$, and especially the re-introduction of the objective function $\Omega$ by using an non-zero $w_{3}$.

Other considerations for future study include non-uniform sensor size in the definition of $\Omega$, nonuniform spatial probability distribution of objects within $\Theta$ and $\Gamma$, and the role of temporal probability in the overall definition of $U$.

\section{REFERENCES}

[1] P. Agarwal and M. Sharir. Arrangements and their Applications, pages 49-119. Elsevier Science B. V., 1999.

[2] P. Asadzadeh, L. Kulik, E. Tanin, and A. Wirth. On optimal arrangements of binary sensors. In COSIT 2011, pages 168-187. Springer-Verlag Berlin Heidelberg, 2011.

[3] J. Aslam, Z. Butler, F. Constantin, V. Crespi, G. Cybenko, and D. Rus. Tracking a moving object with a binary sensor network. In SenSys '03, pages 150-161. ACM, November 2003.

[4] K. Chakrabarty, S. Iyengar, H. Qi, and E. Cho. Coding theory framework for target location in distributed sensor networks. In Information Technology: Coding and Computing, pages 130-134, Las Vegas, NV, April 2001. 
International Journal of Information Sciences and Techniques (IJIST) Vol.3, No.1, January 2013

[5] A. Krause, A. Singh, and C. Guestrin. Near-optimal sensor placements in gaussian processes: Theory, efficient algorithms and empirical studies. Journal of Machine Learning Research, 9:235-284, 2008.

[6] C. Shannon. A mathematical theory of communication. Bell System Technical Journal, 27:379-423 and 623-656, July, October 1948.

[7] B. Sharif and F. Kamalabadi. Optimal sensor array configuration in remote image formation. IEEE Trans. Image Process., 17(2):155-166, February 2008.

[8] N. Shrivastava, R. Mudumbai, U. Madhow, and S. Suri. Target tracking with binary proximity sensors: Fundamental limits, minimal descriptions, and algorithms. In SenSys '06, pages 251-264. ACM, November 2006.

[9] N. Shrivastava, R. Mudumbai, U. Madhow, and S. Suri. Target tracking with binary proximity sensors. ACM Transactions on Sensor Networks, 5(4):30:1-30:33, November 2009.

\section{Authors}

Stephen P. Emmons is a Ph.D. candidate in Computer Science and Engineering at the University of Texas at Arlington. He has had a career developing commercial software products for technical illustration, 3D solids modelling, and wireless sensor networking spanning over 30 years.

Professor Farhad Kamangar received his $\mathrm{Ph} . \mathrm{D}$. in electrical engineering from the University of Texas at Arlington in 1980 and M.S. in electrical engineering from UTA in 1977. He received his B.S. from the University of Tehran, Iran, in 1975. His research interests include image processing, robotics, signal processing, machine intelligence and computer graphics.
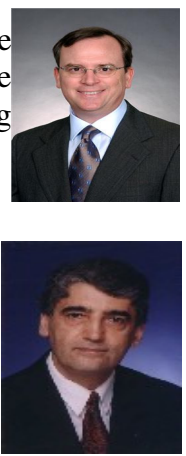